\title{
La relation maître-élève et la question de l'autorité
}

Une étude sur le Bénin

The teacher-pupil relationship and the question of authority. A study of Benin

La relación maestro-alumno y la cuestión de la autoridad

Un estudio sobre Benin

\section{Clarisse Napporn}

\section{(2) OpenEdition}

Journals

Édition électronique

URL : https://journals.openedition.org/ries/5508

DOI : $10.4000 /$ ries.5508

ISSN : 2261-4265

Éditeur

France Education international

Édition imprimée

Date de publication : 1 septembre 2016

Pagination : 57-66

ISBN : 978-2-85420-611-1

ISSN : 1254-4590

Référence électronique

Clarisse Napporn, "La relation maitre-élève et la question de l'autorité ", Revue internationale

d'éducation de Sèvres [En ligne], 72 I septembre 2016, mis en ligne le 01 septembre 2018, consulté le

01 juillet 2021. URL : http://journals.openedition.org/ries/5508 ; DOI : https://doi.org/10.4000/ries. 5508 


\title{
La relation maître-élève et la question de l'autorité
}

\section{Une étude sur le Bénin}

\author{
Clarisse Napporn \\ Université d'Abomey-Calavi
}

L'éducation, dans son sens premier, consiste pour les adultes à créer des conditions permettant aux plus jeunes de développer toutes leurs potentialités. Une bonne part de cette éducation se déroule dans les établissements scolaires, où les enfants sont confiés aux maîtres.

Chacun se souvient de son propre parcours scolaire, de ses relations avec les enseignants et de celles entre les familles et l'école. Conformément à la culture africaine, qui fait de l'âge un critère de valeur, l'autorité de l'enseignant était perçue, au Bénin, comme un prolongement naturel de celle des parents jusqu'en 1990 environ. L'adulte ayant toujours raison, l'autorité exercée par le maître sur l'élève était rarement contestée. Comme l'a montré Cherkaoui à propos de Durkheim (2010), ce rapport « vertical » (Reboul, 2010) tenait au fait que la fonction essentielle de l'institution scolaire était en fait l'éducation morale : l'école devait avant tout viser à donner aux élèves le sens de l'autorité, grâce à des habitudes ancrées dans le respect de la discipline. Pour ce faire, on ne devait pas confier les enfants à des personnes à la morale ou aux mœurs douteuses ou encore n'ayant pas les qualifications requises.

Au Bénin, il est devenu courant, depuis quelques années, d'apprendre par la presse les méfaits de certains enseignants ou l'attitude indélicate de certains parents cherchant à tort à défendre leurs enfants vis-à-vis des enseignants, voire d'élèves suffisamment audacieux pour lever la main sur leurs enseignants.

Le Conseil national de l'éducation (2014), qui a fait de la relation maître-élève l'une de ses préoccupations, évoque des abus de tous ordres: cas de harcèlement moral et sexuel, corruption et d'abus d'autorité sous différentes formes (triche, vente et achat d'épreuves d'examens et de concours, commercialisation de supports de cours et de travaux dirigés, etc.). L'autorité du maître n'est plus systématique et se trouve discutée.

Pour aborder cette question, nous observons le système scolaire en nous limitant au secondaire, en nous appuyant sur une enquête par questionnaire menée par 15 enquêteurs formés auprès de 815 acteurs volontaires du système éducatif dans trois grandes villes (Cotonou, Bohicon, Parakou) : enseignants du primaire et du secondaire, parents d'élèves, représentants d'associations de 
parents d'élèves, élèves, agents administratifs (directeurs pour le primaire et le secondaire, censeurs et surveillants généraux pour le secondaire), secrétaire technique permanent du plan décennal de développement de l'éducation, représentants de syndicats d'enseignants, directeurs départementaux des enseignements primaire et secondaire. Après avoir présenté le contexte béninois et proposé des définitions de l'autorité, nous exposons les différentes formes que prend l'exercice de l'autorité au Bénin et les indicateurs de l'affaiblissement de cette autorité. Les personnes interrogées, si elles ne contestent pas l'autorité, ont des idées sur les modalités de l'exercer.

\section{LE CONTEXTE BÉNINOIS}

Au Bénin, on peut donc dire que la relation maître-élève a subi des mutations. Envisager ce phénomène comme un processus permet de poser l'hypothèse que ces mutations sont tributaires des régimes politiques, des choix effectués et des orientations données au système éducatif.

Il faut remonter aux années 1970 si l'on évoque la question des modalités de recrutement des enseignants, que nous pensons déterminantes. Sous le régime révolutionnaire (1972-1989), pour faire face à l'insuffisance d'enseignants, il avait été institué un service patriotique d'une année, au cours de laquelle des étudiants puis des bacheliers (parfois âgés d'à peine 18 ans) devaient effectuer une mission d'enseignement. Certains furent envoyés dans l'enseignement primaire. L'État recrutait aussi pour le primaire ce qu'on appelait alors les «Jeunes instituteurs révolutionnaires» (JIR), sur la base de leur brevet de fin de premier cycle (BEPC) et sans formation professionnelle préalable. Les missions d'enseignement s'achevèrent en 1984 mais les JIR poursuivirent leur carrière. Si des fléaux tels que la corruption, le harcèlement et les abus d'autorité ont toujours existé, ils ont connu un accroissement au cours de cette période (CNE, 2014).

En 1989, des mouvements sociaux contre le pouvoir en place aboutirent à une année blanche, au cours de laquelle nombre d'élèves et d'enseignants se retrouvèrent livrés à eux-mêmes.

En 1990, une conférence nationale aboutit à l'adoption du régime démocratique. Pour l'éducation, on organisa des états généraux, afin de réorienter le système éducatif. L'approche par compétences fut choisie, de nouveaux programmes furent élaborés pendant que, parallèlement, on fermait les écoles normales. Depuis 1990, il n'y a pas eu de planification rigoureuse en matière de recrutements des enseignants. En 1997, il a été décidé qu'il y aurait deux types de recrutement pour l'enseignement primaire : un concours pour les enseignants contractuels titulaires du brevet et du baccalauréat; un test pour les titulaires du baccalauréat, formés ensuite aux nouveaux programmes. Quant à l'accès aux corps de contrôle (conseillers pédagogiques et inspecteurs), il se fait sur concours et est suivi d'une formation. 
En 2003, pour faire face à la pénurie d'enseignants, les écoles ont recruté localement des enseignants dits communautaires, avec les profils les plus variés (brevet, baccalauréat, mais aussi ouvriers, artisans). Ces derniers sont devenus agents de l'État sur décision politique. D'après l'annuaire statistique du ministère de l'enseignement secondaire, de la formation technique et professionnelle (MESFTPRIJ, 2012), $60 \%$ des enseignants seraient qualifiés, ce qui signifie que $40 \%$ ne le sont pas.

En ce qui concerne l'enseignement secondaire, selon les périodes, les établissements ont recruté des titulaires de CAPES ${ }^{1}$ ou de BAPES, donc ayant reçu une formation professionnelle, ainsi que des diplômés aux niveaux licence et maîtrise. En 2003, les collèges ont dû recruter des vacataires de niveau varié (maîtrise, licence, DUEL, DEUG, DUES ${ }^{2}$ ), qui sont également devenus agents contractuels de l'État par reversement sur décision politique.

Ainsi, seuls $15 \%$ des professeurs de mathématiques et $22 \%$ des professeurs de français ont le profil requis pour enseigner ces disciplines. Les autres enseignants ont des profils variés : des gestionnaires et des géographes enseignent les mathématiques, des psychologues ou des sociologues le français (CNE, 2014).

Cette diversité et cette variété des profils des enseignants, au sein des différents niveaux d'enseignement, présagent de façons différentes, pour chacun, de comprendre ses attributions et d'exercer l'autorité, d'instaurer ou non la confiance.

En principe, les formations comportent des modules de législation scolaire et morale professionnelle (organisation du système éducatif, qualités professionnelles et personnelles requises, droits et devoirs, carrière, sanctions et responsabilités), qui initient le futur enseignant à ses attributions et à ses missions (guide du normalien et de la normalienne). Ceci devrait favoriser le respect du règlement intérieur et de la hiérarchie et éveiller la conscience professionnelle, facilitant ainsi l'exercice de l'autorité.

\section{DÉFINITIONS DE L'AUTORITÉ}

Reboul (2010) définit «l'autorité comme le pouvoir qu'a quelqu'un de faire faire à d'autres ce qu'il veut sans avoir à recourir à la violence, pouvoir dû soit à sa position sociale, soit à sa compétence, soit à son ascendant ». Robbes (2006) propose de l'autorité éducative une triple signification indissociable : être l'autorité (statutaire), avoir de l'autorité (faire grandir l'autre) et faire autorité (autorité de capacité et de compétence). En principe, un enseignant doit pouvoir combiner les trois caractéristiques : il a le pouvoir institutionnel, il doit savoir en user

1. Le CAPES (certificat d'aptitude au professorat de l'enseignement secondaire) est obtenu après un baccalauréat et cinq années de formation, soit une maîtrise et une année de formation professionnelle, soit une licence ou un BAPES et deux années de formation professionnelle ; le BAPES (brevet d'aptitude au professorat de l'enseignement secondaire) s'obtient après une licence suivie d'une année de formation professionnelle ou un baccalauréat et trois années de formation professionnelle.

2. Les DUEL, DEUG, DUES équivalent respectivement à un baccalauréat suivi de deux années d'études en lettres, gestion et sciences. 
parce qu'il a des compétences avérées dans sa discipline mais il en reconnaît aussi les limites pour faire place à l'expression de l'élève, qu'il accompagne vers l'autodiscipline.

Enfin, Prairat (2008) établit une distinction entre l'auctoritas et la potestas:

[La potestas est] le pouvoir fondé sur la fonction, le grade ou le statut. C’est le pouvoir légal, accordé par les instances supérieures de la société (militaires, judiciaires, scolaires par exemple) pour prendre des décisions et commander dans un domaine donné en recourant à la contrainte le cas échéant.

Cette dernière définition décrit bien l'autorité exercée par la plupart des enseignants au Bénin, et notamment leur rapport aux sanctions.

C'est ce sens de potestas qui a en effet été le plus souvent associé à la notion d'autorité par les personnes interrogées au cours de l'enquête. Il est lié à l'organisation de la société : autorité du roi et du chef de village, de clan, de tribu, autorité du dignitaire, du chef religieux, autorité du père, du parent... Il est difficile de s'y soustraire.

L'auctoritas relève davantage de l'influence, de l'ascendant, du crédit. C'est l'art d'obtenir l'adhésion sans recourir à la menace ou la contrainte. Elle recommande plus qu'elle ne commande. Au Bénin, cette forme d'autorité est un idéal. Peu d'enseignants et de responsables incarnent l'auctoritas, qui n'est du reste pas toujours bien comprise.

\section{FORMES ET DÉRIVES DE LA RELATION MAîTRE-ÉLÈVE}

\section{Une enquête empirique nécessaire}

Avant d'aborder les formes de la relation maître-élève, nous apportons quelques précisions sur l'enquête menée, née du souci de disposer de données complémentaires de celles fournies par le CNE. Les questions posées étaient d'ordre varié et relatives à la vie dans l'établissement et dans la classe : pour les élèves, communication, sanctions, confiance, relation avec la hiérarchie et l'administration, écoute, équité dans l'évaluation ; pour les enseignants, respect et liberté d'initiative, climat des apprentissages, dispositions de protection contre les abus, relations entre parents, enseignants et administration, confiance dans l'établissement et dans le système éducatif, évaluation professionnelle ; pour les parents, fonctionnement de l'association des parents d'élèves, réactions par rapport aux sanctions reçues, existence d'un cadre inclusif autour de la question de l'autorité, relations avec les enseignants et avec l'administration; pour les chefs d'établissement et les cadres éducatifs, critères de nomination et relations avec l'autorité de tutelle, avec les parents, moyens et stratégies pour rétablir l'autorité et la confiance dans le système, exercice de l'autorité dans l'établissement, etc. 


\section{Constats sur les dysfonctionnements}

Les données recueillies ont permis de mettre au jour certains comportements d'enseignants, de la maternelle au primaire et au secondaire : violences (verbales, physiques), absence de communication, vente forcée de travaux dirigés et d'épreuves, corruption, retenues abusives, mauvaises notes injustifiées, trafics divers, harcèlement moral et sexuel, etc.

$25 \%$ des apprenants du secondaire enquêtés (soit 37 personnes) évoquent des intimidations, des humiliations publiques et des châtiments corporels. Ils soulignent que l'élève a toujours tort face à l'administration, toutepuissante notamment dans le primaire. Dans le secondaire, où les adolescents peuvent davantage développer une attitude réflexive sur les actes, les paroles et les pensées, les plaintes sont plus fréquentes mais aboutissent rarement, car l'autorité de l'enseignant ne doit pas être remise en cause. Ainsi, ceux qui violentent les enfants sont souvent simplement mutés. À la question de savoir s'il existe un dispositif qui permette aux élèves de se plaindre sans être inquiétés, $25 \%$ du personnel administratif (soit 15 personnes) fournissent les réponses suivantes : écoute des élèves, confidentialité garantie sans représailles, comité conseil de classe (dispositif permettant aux élèves d'échanger entre eux et de se plaindre sans être inquiétés).

Les abus des adultes sont d'ailleurs confirmés par des enseignants du primaire et du secondaire (20\%, soit environ 50 personnes), qui disent avoir recours aux châtiments corporels pour asseoir leur autorité. Des parents (45\% soit 108 personnes) affirment avoir des raisons de déplorer la façon dont les enseignants exercent leur autorité mais avouent ne pas toujours réagir, afin de ne pas porter tort à leurs enfants. $5 \%$ des élèves finissent par considérer l'école comme un milieu hostile et s'y rendent contraints par leurs parents (ils sont âgés de 18 à 21 ans). L'usage de la violence pour asseoir l'autorité (même s'il n'est pas généralisé) est récusé par des parents, des responsables du système éducatif et par les responsables associatifs interrogés, de même que par certains enseignants (environ 500 personnes), qui plaident pour le respect des textes, les châtiments corporels étant interdits.

\section{Hypothèses sur les causes}

Dans les collèges, s'il existe toujours des censeurs et des surveillants généraux (et non des conseillers d'éducation), ils sont parfois en nombre très insuffisant (ainsi, le lycée Béhanzin de Porto Novo, qui accueille environ deux mille élèves, s'étend sur plusieurs hectares et dispose de trois surveillants). L'accompagnement des élèves dans ces conditions ne peut se faire convenablement.

À l'insuffisante qualification des enseignants déjà évoquée plus haut bien souvent, il faut ajouter les effectifs pléthoriques des classes, la moyenne étant de quatre-vingts élèves dans les établissements publics, massification oblige. 
Ces conditions ne permettent pas un bon suivi ni la prise en compte des besoins spécifiques des élèves. Il arrive régulièrement que des élèves en sachent plus que leurs enseignants et ne se taisent pas. La mise en œuvre des programmes demande un bon niveau de formation et de culture des enseignants.

\section{Une confiance déçue}

En effet, « enseigner consiste à la fois à transmettre le goût de la mesure et de la règle à un enfant qui l'ignore »(Cherkaoui, 2010). Cela signifie que l'enseignant doit être conscient de son rôle dans la vie de l'élève. Ce dernier a besoin de règles pour se structurer. Mais il a aussi besoin de les comprendre pour se les approprier.

$70 \%$ des élèves des établissements confessionnels interrogés (40 élèves environ) déclarent admirer leurs enseignants et voir en eux des modèles.

C'est un avis que les parents ne partagent pas vraiment. $75 \%$ d'entre eux (public, privé laïque et confessionnel), soit 180 personnes, déclarent ne pas faire confiance aux enseignants, compte tenu de leurs compétences et de leurs comportements. Ils évoquent la pédagogie et le fait que les enseignants n'encadrent pas bien les enfants. Ils ajoutent qu'ils n'ont pas le choix, à moins de faire changer d'établissement leurs enfants chaque année. On note une certaine ambivalence dans les réponses par rapport aux établissements confessionnels auxquels les parents confient leurs enfants : d'un côté, un attrait pour leur bonne réputation mais de l'autre, des regrets devant certaines attitudes constatées (manque de communication, d'écoute, rôle de figuration de l'association des parents d'élèves). Les religieux joueraient "sur le respect de la soutane ou de la robe».

L'administration des établissements ne fonctionne pas toujours bien, en raison d'une syndicalisation à outrance (on dénombre plus de quatre-vingts syndicats dans l'enseignement primaire et soixante-dix dans l'enseignement secondaire) et d'une politisation, indiquent les responsables administratifs à divers niveaux. Ils évoquent le départ d'enseignants en pleine année pour cause de nomination, de mutation. Deux tiers des 60 responsables interrogés ont parlé de nominations politiques - ne respectant pas les critères prévus pour occuper certaines fonctions.

Le fonctionnement actuel du système éducatif entretient une espèce de confusion en matière de gouvernance et n'inspire pas vraiment confiance aux acteurs, qu'il s'agisse des élèves, des parents et de certains responsables. Ainsi, $75 \%$ des parents interrogés (soit 180 personnes) n'ont confiance ni dans l'organisation, ni dans les contenus, ni dans la gouvernance. Les programmes d'enseignement actuels ont été généralisés dans les établissements depuis 2000, avec la pédagogie de l'approche par les compétences. Les stratégies d'enseignement/ apprentissage sont nouvelles pour les parents, qui peuvent difficilement accompagner leurs enfants. Parallèlement, la plupart des enseignants sont sans formation initiale avec des qualifications discutables. Cela exige des parents un accompagnement et un soutien scolaire accrus (Napporn et Baba-Moussa, 2013). 


\section{Des causes multiples}

Les causes expliquant les dérives de la relation maître-élève et de l'exercice de l'autorité sont multiples. L'autorité n'est pas seulement exercée par l'enseignant sur l'apprenant. L'enseignant (communautaire, vacataire, contractuel de l'État, agent permanent) a lui-même des autorités qui apprécient son travail (directeur, surveillant général, censeur). Il y a ensuite le conseiller pédagogique, l'inspecteur, le directeur départemental de l'enseignement auquel il est rattaché. Pour que le système fonctionne convenablement, il faut que chacun soit à sa place, connaisse ses attributions et accomplisse ses tâches convenablement sans outrepasser ses fonctions. Si l'on installe, par exemple, un simple enseignant au poste d'inspecteur, le système est mis à mal car l'autorité exercée par cette personne ne repose pas sur des bases prévues par la législation et cela crée un malaise. L'affaiblissement de l'autorité est donc généré par le système. Des parents et responsables administratifs enquêtés (environ 220 personnes) citent la personnalité des enseignants, l'impunité, le manque de qualification, la politisation, la syndicalisation, la corruption, l'absence de conscience professionnelle, le mode de recrutement, le rôle des médias, les séries télévisées et les réseaux sociaux.

\section{Crises de l'autorité, de la confiance, perte de repères}

Les données de l'enquête ne permettent pas de distinguer crise de l'autorité et crise de la confiance. Les deux aspects vont de pair et ont plusieurs facettes. Nous pensons qu'il s'agit d'abord d'une crise de la famille, de la société, d'une crise des valeurs morales et civiques, avec une perte de repères. Au Bénin, la crise de confiance entre l'administration et les enseignants est particulièrement visible dans le secteur public. Un chef d'établissement du public (collège) a enregistré, par exemple des départs massifs d'enseignants en cours d'année, ce qui ne favorise pas le fonctionnement de l'établissement. Ces départs sont dus à des nominations ou des mutations en milieu d'année scolaire. L'administration entrave elle-même le bon fonctionnement des établissements sous sa tutelle...

$25 \%$ des parents enquêtés (soit 60 personnes) révèlent aussi un mauvais fonctionnement des associations de parents d'élèves dans les établissements (soit ils en ignorent l'existence, soit elles ne jouent pas un rôle prépondérant, soit elles interviennent de façon subjective). Ils ajoutent que certains parents restent membres des associations, alors qu'ils n'ont plus d'enfants dans l'établissement depuis longtemps. Il est donc parfois difficile de déterminer quel rôle joue vraiment cette instance, dans certains cas. 


\section{Se préoccuper de la qualité de la relation maître-élève}

Si la plupart des institutions et des grandes réflexions en matière d'éducation insistent fortement sur la qualification des enseignants et la revalorisation de la fonction enseignante, on se préoccupe très peu de la qualité de la relation maître-élève, jugée pourtant insatisfaisante par de nombreux acteurs au Bénin. $60 \%$ des parents enquêtés (144 personnes) pensent que l'école actuelle dégrade l'éducation donnée à la maison et que les enseignants ne maîtrisent ni la pédagogie ni leur discipline.

En principe, des qualités humaines, pédagogiques, intellectuelles et morales sont attendues des enseignants. Si nous considérons que les qualités intellectuelles et la qualification professionnelle sont obligatoires, les autres qualités ne doivent pas être attendues d'emblée. Mais les enseignants se doivent d'en être informés, et de travailler à les acquérir et à les cultiver dans l'exercice de leur fonction.

Les parents ( $40 \%$ ) et les élèves ( $5 \%$, âgés de 18 à 21 ans) interrogés sur l'ambiance des apprentissages et le cadre global de l'école souhaitent des approches pédagogiques qui valorisent l'apprenant et sa créativité, alors que tous les enseignants ont indiqué ne prendre des initiatives que lorsqu'elles sont validées par la hiérarchie. D'ailleurs, la démarche d'enseignement-apprentissage est la même dans tous les établissements publics. Les établissements privés s'autorisent le recours à des outils complémentaires (matériels didactiques, renforcements des capacités des enseignants). Nous partageons l'avis de quelques parents (5\% soit 12 parents) qui veulent que l'on repense les valeurs et que l'on communique avec les enfants, que les enseignants et responsables administratifs expliquent suffisamment le contenu des règlements intérieurs des établissements. Ils souhaitent que les enseignants fassent preuve de cohérence et soient des modèles, des guides pour leurs élèves, conditions nécessaires pour leur faire confiance.

En 2014, un forum sur l'éducation a été organisé pour repenser le système éducatif béninois, qui fait face à de nombreux défis. Le nouveau gouvernement nommé en avril 2016 a mis en place un comité pour la réforme du système éducatif, en commençant par les aspects administratifs : il s'agit de restructurer l'architecture de l'école et d'instaurer un mode de gouvernance et de fonctionnement cohérent.

Les programmes en vigueur exigent des enseignants qu'ils soient des accompagnateurs, des médiateurs, des facilitateurs plutôt que des maîtres qui imposent ou écrasent de façon autoritariste, puisqu'une place est faite au travail de l'élève (travail individuel, en groupe avec les pairs et synthèse avec l'enseignant). Si en effet «favoriser les pratiques d'enseignement qui maintiennent la confiance des élèves constitue un défi éducatif » (Galland, 2015), c'est vers ce modèle qu'il faut tendre. 
La question de l'autorité ne peut être évacuée de la relation éducative ou de la relation maître-élève. Il n'est pas nécessaire qu'elle se décline comme autrefois, c'est-à-dire dans une conception autoritariste, qui ne convient plus vraiment. Aujourd'hui, au Bénin, il est prescrit par les textes officiels que les élèves soient coauteurs et acteurs de la construction de leurs connaissances. Cela signifie que le rapport à l'adulte et à l'autorité se modifie. Il doit permettre aux différents acteurs (administration, parents, enseignants, élèves) de collaborer et de coopérer en toute confiance, chacun dans ses fonctions. Pour ce faire, nous pensons qu'il leur faut communiquer, se parler et se comprendre. Mialaret (2014) écrit à juste titre que le processus éducatif est essentiellement un processus de communication.

L'autorité est un princeps originaire spécifique de l'espèce humaine, trait évolutif sélectionné comme principe régulateur devenu caractéristique de la relation entre un adulte et son enfant, indispensable à sa survie et à son inscription dans le groupe social... La communication corporelle y a une place essentielle. La relation d'autorité se construit donc sur des modalités de communication. (Robbes, 2006)

Cette définition de Marcelli proposée par Robbes prend tout son sens à nos yeux : sans communication, il ne saurait y avoir de véritable relation d'autorité. Il est donc difficile d'exercer l'autorité sans communiquer. Les caractéristiques que Prairat (2008) reconnaît à l'influence éducative de l'auctoritas deviennent pertinentes. Elle est libératrice, parce qu'elle vise à faire grandir, et indirecte, parce qu'elle doit suggérer plus qu'imposer, et respecter la graduation des étapes pour l'acquisition de l'autonomie et du sens de la responsabilité. Enfin, elle est temporaire, car on n'exerce pas indéfiniment son autorité. Nous avons apprécié l'exercice de l'autorité avant l'ère démocratique parce que les règles de fonctionnement étaient connues. Il est toujours bien, lorsqu'on s'inscrit dans un processus, d'en connaître les règles, même si l'on est un enfant.

Reboul (2010) distingue six figures de l'autorité : le contrat, l'expert, l'arbitre, le modèle, le leader et le Roi-Père. Si l'on se réfère aux propos tenus par les parents, les élèves et les responsables interrogés dans notre enquête, les trois figures souhaitées sont celles du contrat, de l'expert et de l'arbitre.

Il est d'autant plus évident qu'il faut envisager d'autres modes d'exercice de l'autorité éducative que, dans les familles elles-mêmes, la relation éducative a subi des modifications.

Selon Cherkaoui (2010), le maître tire son autorité non de lui-même mais de sa fonction. De par son statut, il doit faire respecter des règles institutionnelles. Fournier (2011) affirme qu'il n'existe ni secret ni recette, mais un patchwork de pratiques professionnelles.

[Une] autorité co-construite ou négociée (...) passe par le changement des représentations de l'enseignement, la reconnaissance des élèves en tant qu'acteurs capables d'autonomie mais surtout que l'école dispose de ressources pour œuvrer dans ce sens. (Tenaerts, 2008) 
Nous souhaitons avec Tavoillot (2015) que l'autorité du maître soit réfléchie, limitée et "de service » : en un mot, démocratique. Ce qui ne signifie pas que chacun doive faire ce qu'il veut. La démocratie implique une certaine discipline. Au Bénin, le chemin est encore long.

\section{BiBLIOGRAPHIE}

CHERKaOUi M. (2010): Sociologie de l'éducation, Paris, PUF (QSJ), n 2270, $8^{e}$ édition.

Conseil national de l'éducation (2014): Ateliers thématiques en préparation au II Forum national de l'éducation, Cotonou.

FORAY P. (2009) : "Les trois formes de l'autorité scolaire ", Le Télémaque 1/2009 $\mathrm{n}^{\circ} 35$, p. 73-86 [en ligne], www.cairn.info/revue-le-telemaque-2009-1-page-73.htm, DOI : $10.3917 /$ tele.035.0073.

FOURNIER M. (2011) : "Autorité : où sont passés les modèles ? ", dans Éduquer et former, Auxerre, éditions Sciences Humaines.

GALAND B. (2015) : "À l'école de l'estime de soi », Sciences humaines n 271.

MIALARET G. (2014) : Psychologie de l'éducation, Paris, PUF (QSJ) nº 3475, 3e éd.

Ministères en charge de l'éducation (2007) : Actes du $1^{\text {er }}$ forum, Cotonou, Quadriservices.

Ministère de l'enseignement secondaire de la formation technique et professionnelle, de la reconversion et l'insertion des jeunes (MESFTPRIJ) : Annuaire statistique 2012. NAPPORN C., BABA-MOUSSA A.R. (2013): "Accompagnement et soutien scolaires : l'expérience béninoise ", Revue internationale d'éducation de Sèvres, $\mathrm{n}^{\circ} 62$, avril, p. 79-88.

REBOUL O. (2010): La philosophie de l'éducation, Paris, PUF (QSJ), n 2441, $10^{\mathrm{e}}$ édition.

ROBBES B. (2006) : "Les trois conceptions actuelles de l'autorité ", Cahiers pédagogiques, [en ligne] www.cahiers-pédagogiques.com/les trois-conceptions-actuellesde-l-autorité, 28 mars, consulté le 13 mars 2016.

TAVOILlOT P.-H. (2015) : "Peut-on se passer d'autorité?», Sciences humaines, $\mathrm{n}^{\circ} 266$, janvier.

TENAERTS M.-N. (2008) : "Société et éducation en crise d'“autorité” ", [en ligne] http://www.ufapec.be/files/files/crise\%20d'autorité.pdf, 6 pages, consulté le 10 avril 2016.

USAID-Bénin, Ministère des enseignements primaire et secondaire, Guide du normalien et de la normalienne (non daté). 